\title{
The Magellanic Clouds and the Primordial Helium Abundance
}

\author{
Manuel Peimbert and Antonio Peimbert \\ Instituto de Astronomía, Universidad Nacional Autónoma de México. \\ Apdo. Postal 70-264, México, D.F. 04510
}

\begin{abstract}
A new determination of the pregalactic helium abundance based on the Magellanic Clouds H II regions is discussed. This determination amounts to $Y_{p}=0.2345 \pm 0.0030$ and is compared with those derived from giant extragalactic $\mathrm{H}$ II regions in systems with extremely low heavy elements content. It is suggested that the higher primordial value derived by other authors from giant $\mathrm{H}$ II region complexes could be due to two systematic effects: the presence of neutral hydrogen inside the helium Strömgren sphere and the presence of temperature variations inside the observed volume.
\end{abstract}

\section{Introduction}

The determination of the pregalactic, or primordial, helium abundance by mass $Y_{p}$ is paramount for the study of cosmology, the physics of elementary particles, and the chemical evolution of galaxies (e. g. Fields \& Olive 1998, Izotov et al. 1999, Peimbert \& Torres-Peimbert 1999, and references therein). In this review we briefly discuss the method used to derive $Y_{p}$ and its main sources of error as well as a new determination based on observations of the SMC. This determination is compared with those carried out earlier based on extremely metal poor extragalactic H II regions.

The Magellanic Clouds determination of $Y_{p}$ can have at least four significant advantages and one disadvantage with respect to those based on distant H II region complexes: a) no underlying absorption correction for the helium lines is needed because the ionizing stars can be excluded from the observing slit, b) the determination of the helium ionization correction factor can be estimated by observing different lines of sight of a given $\mathrm{H}$ II region, c) the accuracy of the determination can be estimated by comparing the results derived from different points in a given $\mathrm{H}$ II region, d) the electron temperature is generally smaller than those of metal poorer $\mathrm{H}$ II regions reducing the effect of collisional excitation from the metastable $2^{3} \mathrm{~S}$ level of $\mathrm{He} \mathrm{I}$, and e) the disadvantage is that the correction due to the chemical evolution of the SMC is in general larger than for the other systems. 


\section{2. $\mathrm{He}^{+} / \mathrm{H}^{+}$Determinations}

To derive accurate $\mathrm{He}^{+} / \mathrm{H}^{+}$values we need very accurate $N_{e}$ (He II), $T_{e}$ (He II), and $\tau(3889, \mathrm{He} \mathrm{I})$ values. Good approximations to determine $\mathrm{He}^{+} / \mathrm{H}^{+}$for $N_{e}<$ $300 \mathrm{~cm}^{-3}, 13000 \mathrm{~K}<T_{e}<20000 \mathrm{~K}$ and $\tau(3889)=0.0$, have been presented for the main helium lines by Benjamin, Skillman and Smits (1999):

$$
\begin{aligned}
\frac{N\left(\mathrm{He}^{+}\right)}{N\left(\mathrm{H}^{+}\right)} & =\frac{I(6678)}{I(\mathrm{H} \beta)} 2.58 T_{4}^{0.249-2.0 \times 10^{-4} N_{e}} \\
& =\frac{I(4471)}{I(\mathrm{H} \beta)} 2.01 T_{4}^{0.127-4.1 \times 10^{-4} N_{e}} \\
& =\frac{I(5876)}{I(\mathrm{H} \beta)} 0.735 T_{4}^{0.230-6.3 \times 10^{-4} N_{e}}
\end{aligned}
$$

Fortunately for giant extragalacic H II regions $\tau(3889)$ is very small and frequently close to zero. $\tau(3889)$ can be estimated together with $N_{e}$ (He II) from the $3889 / 4471$ and the $7065 / 4471$ ratios computed by Robbins (1968).

Most authors assume that $T_{e}(\mathrm{O}$ III $)=T_{e}(\mathrm{He}$ II $)$, there are two reasons for this assumption the $\mathrm{He}^{+}$and $\mathrm{O}^{++}$emission regions occupy similar volumes (but not identical ones) and $T_{e}(\mathrm{O}$ III) is easy to measure. Nevertheless the assumption is correct only for isothermal nebulae, and not for real nebulae if very accurate abundances are needed. In the presence of temperature variations along the line of sight the [O III] lines originate preferentially in the high temperature zones and the helium and hydrogen lines in the low temperature zones (e.g. Peimbert $1967,1995)$. It can be shown that the temperature derived from the ratio of the Balmer continuum to a Balmer emission line, $T_{e}(\mathrm{Bac})$, is similar to $T_{e}(\mathrm{He} \mathrm{II})$, and that both temperatures are smaller than $T_{e}(\mathrm{O}$ III).

From models computed with CLOUDY (Ferland 1996) it is found that $T_{e}(\mathrm{Bac})$ - labeled $T_{e}(\mathrm{Hth})$ by CLOUDY - is about $5 \%$ smaller than $T_{e}(\mathrm{O}$ III $)$. Moreover in the model with a homogeneous sphere of I $\mathrm{Zw} 18$ by Stasinska and Schaerer (1999) it is found that $\left\langle T_{e}(\mathrm{Ar}\right.$ III $\left.)\right\rangle=16300 \mathrm{~K}$ (for $63 \%$ of the volume) and $\left\langle T_{e}(\mathrm{Ar}\right.$ IV) $\rangle=18300 \mathrm{~K}$ (for $36 \%$ of the volume) indicating the presence of temperature variations. Notice that the average temperature has to be weighted by the emissivities strengthening the effect of the temperature variations. Moreover there is additional evidence that indicates that the temperature variations are even higher than those predicted by photoionization models (e. g. Peimbert 1995; Luridiana, Peimbert, \& Leitherer 1999; Stasinska and Schaerer 1999). From equations (1-3) it follows that the smaller the adopted temperature the smaller the derived $\mathrm{He}^{+} / \mathrm{H}^{+}$value.

Due to the presence of very strong density variations inside gaseous nebulae the different methods to derive the density yield very different values. The root mean square density, $N_{e}$ (rms), is usually obtained from the observed flux in a Balmer line and by assuming a spherical geometry; $N_{e}(\mathrm{rms})$ provides a minimum value for $N_{e}(\mathrm{He}$ II), the local density needed to derive the helium abundance. Forbidden line ratios of lines of similar excitation energy give us an average density for cases where the density is similar or smaller than the critical density for collisional deexcitation; available line ratios in the visual region are those of 
[S II], [O II], [Cl III], and [Ar IV], unfortunately giant extragalactic H II regions are close to the low density limit of these ratios, the line intensities of [Cl III] are very faint, for most observations of the [O II] lines the resolution is not high enough to separate $\lambda 3726$ from $\lambda 3729$ nor $\lambda 4711$ of [Ar IV] from $\lambda 4713$ of He I. Consequently most of the densities in the literature are those derived from the [S II] lines, but as rightly mentioned by Izotov, Thuan, \& Lipovetsky (1994, 1997) they are not representative of the regions where the He I lines originate. The self-consistent method, advocated by Izotov and collaborators, is based only on line ratios of helium $I$ and is the best method to derive the density of the He II zone: $N_{e}$ (He II).

The stellar underlying absorption can affect the derived He I and $\mathrm{H}$ I emission line intensities and has to be taken into account. The best way to reduce this effect is to avoid the presence of bright early type stars in the observed slit, this can only be done for objects inside the Galaxy and the Magellanic Clouds. To minimize the effect of the underlying absorption it is recommended to use only objects where the line intensities show very large equivalent widths in emission, and to increase the spectral resolution. The best way to correct for underlying absorption is to use starburst models that predict the underlying stellar spectrum. The correction for underlying absorption can be tested by comparing the higher order Balmer lines, that are most affected by this effect, with the brightest Balmer lines that are the least affected. Similarly the underlying absorption effect is larger for $\lambda 4471$ and smaller for $\lambda \lambda 5876,6678$ and 7065 ; the effect for these three lines is in general negligible due to a combination of causes (mainly their large equivalent widths in emission).

\section{Ionization Structure}

The total $\mathrm{He} / \mathrm{H}$ value is given by:

$$
\begin{aligned}
\frac{N(\mathrm{He})}{N(\mathrm{H})} & =\frac{N\left(\mathrm{He}^{0}\right)+N\left(\mathrm{He}^{+}\right)+N\left(\mathrm{He}^{++}\right)}{N\left(\mathrm{H}^{0}\right)+N\left(\mathrm{H}^{+}\right)}, \\
& =I C F(\mathrm{He}) \frac{N\left(\mathrm{He}^{+}\right)+N\left(\mathrm{He}^{++}\right)}{N\left(\mathrm{H}^{+}\right)} .
\end{aligned}
$$

The $\mathrm{He}^{++} / \mathrm{H}^{+}$ratio can be obtained directly from the $4686 / \mathrm{H} \beta$ intensity ratio. In objects of low degree of ionization the presence of neutral helium inside the $\mathrm{H}$ II region is important and $I C F(\mathrm{He})$ becomes larger than 1 . The $I C F(\mathrm{He})$ can be estimated by observing a given nebula at different lines of sight since $\mathrm{He}^{0}$ is expected to be located in the outer regions. Another way to deal with this problem is to observe $\mathrm{H}$ II regions of high degree of ionization where the $\mathrm{He}^{0}$ amount is expected to be negligible. Vílchez \& Pagel (1988) (see also Pagel et al. 1992) defined a radiation softness parameter given by

$$
\eta=\frac{N\left(\mathrm{O}^{+}\right) N\left(\mathrm{~S}^{++}\right)}{N\left(\mathrm{~S}^{+}\right) N\left(\mathrm{O}^{++}\right)}
$$

for large values of $\eta$ the amount of neutral helium is significant, while for low values of $\eta$ it is negligible. 


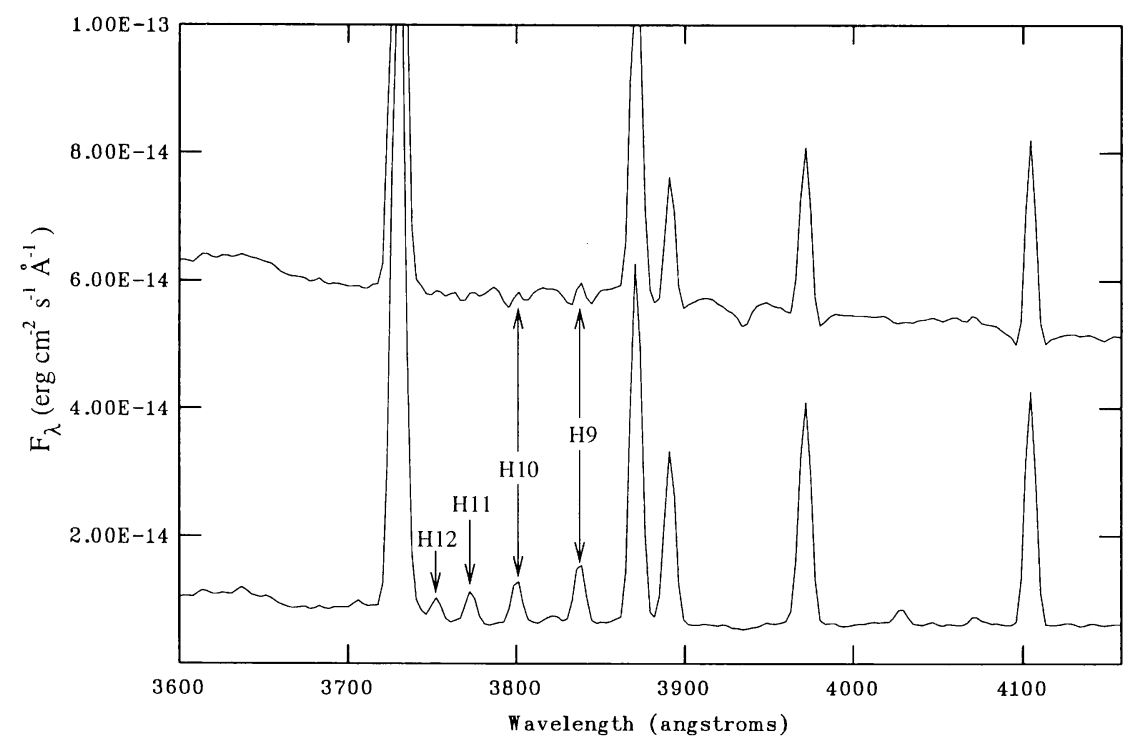

Figure 1. Spectra of NGC 346 with and without underlying absorption. The vertical scale is for the lower spectrum (region 346-A). The flux of the upper spectrum (region 346-B) was normalized to the $\mathrm{H} \alpha$ emission line flux of the lower spectrum.

On the other hand for ionization bounded objects of very high degree of ionization the amount of $\mathrm{H}^{0}$ inside the $\mathrm{He}^{+}$Strömgren sphere becomes significant and the $I C F(\mathrm{He})$ can become smaller than 1 . This possibility was firstly mentioned by Shields (1974) and studied extensively by Armour et al. (1999) for constant density models. Since $\mathrm{H}^{0}$ will be located in a thin shell in the border of the nebula it can be shown that for ionization bounded nebulae of homogeneous density the fraction of $\mathrm{H}^{0} / \mathrm{He}^{+}$will be three times larger for an observation of the whole object than for an observation of a line of sight that includes the center. Similarly this fraction becomes higher than a factor of three for models with decreasing density from the center outwards. Alternatively for density bounded nebulae this effect can be neglected.

\section{SMC}

Peimbert, Peimbert, \& Ruiz (2000a) presented long slit observations of the most luminous H II region in the SMC: NGC 346. They divided the two long slit positions into thirteen areas, four including the brightest stars $(m \sim 14)$ and 9 without stars brighter than $m=17$. In the upper part of Figure 1 we present a spectrum that includes all the observed areas (region 346-B); while in the lower part of Figure 1 we present a spectrum made by the seven brightest areas that do not include the brightest stars (region 346-A).

After correcting region 346-A for extinction, based on the four brightest Balmer lines, it is found that the weaker Balmer lines (H9 to H12) are not 
affected by stellar underlying absorption (see Figure 1), therefore the He lines are not expected to be affected by underlying absorption. The extinction derived from the Balmer lines is in very good agreement with the extinction derived from the stellar cluster by Massey, Parker, \& Garmany (1989), another indication that the stellar underlying absorption of the Balmer lines is negligible.

Table 1. $\quad N\left(\mathrm{He}^{+}\right) / N\left(\mathrm{H}^{+}\right)^{a}$ and $\chi^{2}$ for NGC 346

\begin{tabular}{|c|c|c|c|c|c|}
\hline \multirow[t]{2}{*}{$T_{e}(\mathrm{~K})$} & \multicolumn{5}{|c|}{$N_{e}\left(\mathrm{~cm}^{-3}\right)$} \\
\hline & 53 & 100 & 143 & 162 & 247 \\
\hline 11200 & $\begin{array}{c}805 \\
(83.2)\end{array}$ & $\begin{array}{c}798 \\
(47.7)\end{array}$ & $\begin{array}{c}793 \\
(26.4)\end{array}$ & $\begin{array}{c}791 \\
(20.0)\end{array}$ & $\begin{array}{c}781^{b} \\
(8.24)^{c}\end{array}$ \\
\hline 11800 & $\begin{array}{c}806 \\
(38.6)\end{array}$ & $\begin{array}{c}799 \\
(15.9)\end{array}$ & $\begin{array}{c}793 \\
(7.37)\end{array}$ & $\begin{array}{c}\mathbf{7 9 0} \\
(6.59)\end{array}$ & $\begin{array}{c}780 \\
(20.4)\end{array}$ \\
\hline 11950 & $\begin{array}{c}806 \\
(30.8)\end{array}$ & $\begin{array}{c}799 \\
(11.7)\end{array}$ & $\begin{array}{c}793 \\
(6.53)^{d}\end{array}$ & $\begin{array}{c}790 \\
(7.25)\end{array}$ & $\begin{array}{c}779 \\
(27.7)\end{array}$ \\
\hline 12400 & $\begin{array}{c}807 \\
(15.0)\end{array}$ & $\begin{array}{c}\mathbf{7 9 9} \\
(7.17)\end{array}$ & $\begin{array}{c}793 \\
(12.5)\end{array}$ & $\begin{array}{c}790 \\
(17.9)\end{array}$ & $\begin{array}{c}778 \\
(58.6)\end{array}$ \\
\hline 13000 & $\begin{array}{c}809 \\
(9.72)\end{array}$ & $\begin{array}{c}800 \\
(18.2)\end{array}$ & $\begin{array}{c}793 \\
(38.4)\end{array}$ & $\begin{array}{c}790 \\
(50.2)\end{array}$ & $\begin{array}{c}777 \\
(118)\end{array}$ \\
\hline
\end{tabular}

a Given in units of $10^{-4}, \chi^{2}$ values in parenthesis.

$b$ The $\mathrm{He}^{+} / \mathrm{H}^{+}$values in boldface correspond to the minimum $\chi^{2}$ values at a given temperature.

c The minimum $\chi^{2}$ value at a given temperature is presented in italics.

$d$ The smallest $\chi^{2}$ value for all temperatures and densities is presented in boldface, thus defining $T_{e}\left(\mathrm{He}\right.$ II) and $N_{e}$ (He II).

To derive the $\mathrm{He}^{+} / \mathrm{H}^{+}$value, in addition to the Balmer lines, we made use of nine He I lines, $\lambda \lambda 3889,4026,4387,4471,4921,5876,6678,7065$, and 7281 to determine $N_{e}$ (He II) and $T_{e}$ (He II) self-consistently. In Table 1 we present $\mathrm{He}^{+} / \mathrm{H}^{+}$values for different temperatures and densities; the temperatures were selected to include $T_{e}\left(\mathrm{O}\right.$ III), $T_{e}(\mathrm{Bac}), T_{e}(\mathrm{He} \mathrm{II})$, and two representative temperatures; the densities were selected to include the minimum $\chi^{2}$ at each one of the five temperatures. The temperature with the minimum $\chi^{2}$ is the self-consistent $T_{e}(\mathrm{He} \mathrm{II})$ and amounts to $11950 \pm 560 \mathrm{~K}$; this temperature is in excellent agreement with the temperature derived from the Balmer continuum that amounts to $11800 \pm 500 \mathrm{~K}$, alternatively $T_{e}(\mathrm{O}$ III) amounts to $13070 \pm 100 \mathrm{~K}$. Notice that the $\chi^{2}$ test requires a higher density for a lower temperature, increasing 
the dependence on the temperature of the $\mathrm{He}^{+} / \mathrm{H}^{+}$ratio. The values in Table 1 correspond to the case where $\tau$ (3889) equals zero, for higher values of $\tau$ (3889) the $\chi^{2}$ values increase. In Table 1 the $\mathrm{He}^{+} / \mathrm{H}^{+}$values in boldface and the $\chi^{2}$ values in italics correspond to the minimum value of $\chi^{2}$ at a given $T_{e}$, the $\chi^{2}$ value in boldface is the smallest value for all temperatures and all densities.

From Table 1 we obtain that $T_{e}(\mathrm{He}$ II $)=11950 \mathrm{~K}$ and $N_{e}(\mathrm{He} \mathrm{II})=143 \mathrm{~cm}^{-3}$, which correspond to $\mathrm{He}^{+} / \mathrm{H}^{+}=0.0793 \pm 0.007$. By comparing the $\mathrm{He} / \mathrm{H}$ values for lines of sight with different ionization degree it is found that $I C F(\mathrm{He})=1.00$. To obtain the total $\mathrm{He} / \mathrm{H}$ value we have added the contribution of $\mathrm{He}^{++} / \mathrm{H}^{+}$ that amounts to $2.2 \times 10^{-4}$.

In Table 2 we present the helium abundance by mass $Y$ (SMC), derived from NGC 346. The $Y(\mathrm{SMC})$ values were derived from Table 1 , the $\mathrm{He}^{++} / \mathrm{H}^{+}$values and the $Z$ values determined by Peimbert et al. (2000a).

Table 2. $\quad Y(\mathrm{SMC})$

\begin{tabular}{cccccc}
\hline$T_{e}(\mathrm{~K})$ & \multicolumn{5}{c}{$N_{e}\left(\mathrm{~cm}^{-3}\right)$} \\
& 53 & 100 & 143 & 162 & 247 \\
\hline 11200 & 0.2431 & 0.2416 & 0.2404 & 0.2399 & $\mathbf{0 . 2 3 7 7}^{a}$ \\
11800 & 0.2435 & 0.2419 & 0.2405 & $\mathbf{0 . 2 3 9 9}$ & 0.2375 \\
11950 & 0.2436 & 0.2420 & $\mathbf{0 . 2 4 0 5}$ & 0.2399 & 0.2374 \\
12400 & 0.2439 & $\mathbf{0 . 2 4 2 1}$ & 0.2406 & 0.2399 & 0.2372 \\
13000 & $\mathbf{0 . 2 4 4 3}$ & 0.2423 & 0.2407 & 0.2400 & 0.2370 \\
\hline \hline
\end{tabular}

${ }^{a}$ Boldface values correspond to minimum $\chi^{2}$ values, see Table 1 .

\section{5. $Y_{p}$}

To determine the $Y_{p}$ value from the SMC it is necessary to estimate the fraction of helium present in the interstellar medium produced by galactic chemical evolution. We will assume that

$$
Y_{p}=Y(\mathrm{SMC})-Z(\mathrm{SMC}) \frac{\Delta Y}{\Delta Z} .
$$

Peimbert et al. (2000a) find that for $T_{e}(\mathrm{He} \mathrm{II})=11950 \pm 500 \mathrm{~K}, Y(\mathrm{SMC})=$ $0.2405 \pm 0.0018$ and $Z(\mathrm{SMC})=0.00315 \pm 0.00029$. To estimate $\Delta Y / \Delta Z$ we will consider three observational determinations and a few determinations predicted by chemical evolution models.

Peimbert, Torres-Peimbert, \& Ruiz (1992) and Esteban et al. (1999) found that $Y=0.2797 \pm 0.006$ and $Z=0.0212 \pm 0.003$ for the Galactic H II region M17, where we have added $0.10 \mathrm{dex}$ and $0.08 \mathrm{dex}$ to the carbon and oxygen gaseous abundances to take into account the fraction of these elements embedded in dust grains (Esteban et al. 1998). By comparing the $Y$ and $Z$ values of M17 with those of NGC 346 (Peimbert et al. 2000a) we obtain $\Delta Y / \Delta Z=2.17 \pm$ 
0.4. M17 is the best $\mathrm{H}$ II region to determine the helium abundance because among the brightest galactic H II regions it is the one with the highest degree of ionization and consequently with the smallest correction for the presence of $\mathrm{He}^{0}$ (i.e. $I C F(\mathrm{He})$ is very close to unity). It can be argued that the M17 values are not representative of irregular galaxies, on the other hand they provide the most accurate observational determination. From a group of 10 irregular and blue compact galaxies, that includes the LMC and the SMC, Carigi et al. (1995) found $\Delta Y / \Delta Z=2.4 \pm 0.6$, where they added 0.2 dex to the $\mathrm{O} / \mathrm{H}$ abundance ratios derived from the nebular data to take into account the temperature structure of the $\mathrm{H}$ II regions and the fraction of $\mathrm{O}$ embedded in dust, moreover they also estimated that $\mathrm{O}$ constitutes $54 \%$ of the $Z$ value. Izotov \& Thuan (1998) from a group of 45 supergiant $H$ II regions of low metallicity derived a $\Delta Y / \Delta Z=$ $2.3 \pm 1.0$; we find from their data that $\Delta Y / \Delta Z=1.5 \pm 0.6$ by adding 0.2 dex to the $\mathrm{O}$ abundances to take into account the temperature structure of the $\mathrm{H}$ II regions and the fraction of $\mathrm{O}$ embedded in dust.

Based on their two-infall model for the chemical evolution of the Galaxy Chiappini, Matteucci, \& Gratton (1997) find $\Delta Y / \Delta Z=1.6$ for the solar vicinity. Carigi (2000) computed chemical evolution models for the Galactic disk, under an inside-out formation scenario, based on different combinations of seven sets of stellar yields by different authors; the $\Delta Y / \Delta Z$ spread predicted by her models is in the 1.2 to 1.9 range for the Galactocentric distance of M17 (5.9 kpc).

Carigi et al. (1995), based on yields by Maeder (1992), computed closed box models adequate for irregular galaxies, like the SMC, and obtained $\Delta Y / \Delta Z=$ 1.52. They also computed models with galactic outflows of well mixed material, that yielded $\Delta Y / \Delta Z$ values similar to those of the closed box models, and models with galactic outflows of O-rich material that yielded values higher than 1.52. The maximum $\Delta Y / \Delta Z$ value that can be obtained with models of O-rich outflows, without entering into contradiction with the $\mathrm{C} / \mathrm{O}$ and $(Z-\mathrm{C}-\mathrm{O}) / \mathrm{O}$ observational constraints, amounts to 1.69 .

Carigi, Colín, \& Peimbert, (1999), based on yields by Woosley, Langer, $\&$ Weaver (1993) and Woosley \& Weaver (1995), computed chemical evolution models for irregular galaxies also, like the SMC, and found very similar values for closed box models with bursting star formation and constant star formation rates that amounted to $\Delta Y / \Delta Z=1.71$. The models with O-rich outflows can increase the $\Delta Y / \Delta Z$, but they predict higher $\mathrm{C} / \mathrm{O}$ ratios than observed.

From the previous discussion it follows that $\Delta Y / \Delta Z=1.9 \pm 0.5$ is a representative value for models and observations of irregular galaxies. Moreover, this value is in good agreement with the models and observed values of the disk of the Galaxy.

The $Y_{p}$ values in Table 3 were computed by adopting $\Delta Y / \Delta Z=1.9 \pm 0.5$. The differences between Tables 2 and 3 depend on $T_{e}$ because the lower the $T_{e}$ value the higher the $Z$ value for the SMC.

\section{Discussion}

The $Y_{p}$ value derived by us is significantly smaller than the value derived by Izotov \& Thuan (1998) from the $Y-\mathrm{O} / \mathrm{H}$ linear regression for a sample of 45 BCGs, and by Izotov et al. (1999) from the average for the two most metal 
Table 3. $\quad Y_{p}$ Derived from the SMC

\begin{tabular}{cccccc}
\hline$T_{e}(\mathrm{~K})$ & \multicolumn{5}{c}{$N_{e}\left(\mathrm{~cm}^{-3}\right)$} \\
& 53 & 100 & 143 & 162 & 247 \\
\hline 11200 & 0.2363 & 0.2348 & 0.2336 & 0.2331 & $\mathbf{0 . 2 3 0 9}^{a}$ \\
11800 & 0.2373 & 0.2357 & 0.2343 & $\mathbf{0 . 2 3 3 7}$ & 0.2313 \\
11950 & 0.2376 & 0.2360 & $\mathbf{0 . 2 3 4 5}$ & 0.2339 & 0.2314 \\
12400 & 0.2384 & $\mathbf{0 . 2 3 6 6}$ & 0.2351 & 0.2344 & 0.2317 \\
13000 & $\mathbf{0 . 2 3 9 5}$ & 0.2375 & 0.2359 & 0.2352 & 0.2322 \\
\hline \hline
\end{tabular}

${ }^{a}$ Boldface values correspond to minimum $\chi^{2}$ values, see Table 1 .

deficient galaxies known (I Zw 18 and SBS 0335-052), that amount to $0.2443 \pm$ 0.0015 and $0.2452 \pm 0.0015$ respectively.

The difference could be due to systematic effects in the abundance determinations. There are two systematic effects not considered by Izotov and collaborators that we did take into account, the presence of $\mathrm{H}^{0}$ inside the $\mathrm{He}^{+}$region and the use of a lower temperature than that provided by the [O III] lines. We consider the first effect to be a minor one and the second to be a mayor one but both should be estimated for each object.

From constant density chemicaly homogeneous models computed with CLOUDY we estimate that the maximum temperature that should be used to determine the helium abundance should be $5 \%$ smaller than $T_{e}(\mathrm{O}$ III). Moreover, if there is additional energy injected to the $\mathrm{H}$ II region $T_{e}$ (He II) should be even smaller.

Luridiana, Peimbert, \& Leitherer (1999) produced a detailed photoionized model of NGC 2363. For the slit used by Izotov, Thuan, \& Lipovetsky (1997) they find an $I C F(\mathrm{He})=0.993$; moreover they also find that the $T_{e}(\mathrm{O}$ III $)$ predicted by the model is considerably smaller than observed. From the data of Izotov et al. (1997) for NGC 2363, adopting a $T_{e}$ (He II) $10 \%$ smaller than $T_{e}(\mathrm{O}$ III $)$ and $\Delta Y / \Delta Z=1.9 \pm 0.5$ we find that $Y_{p}=0.234 \pm 0.006$.

Similarly, Stasinska \& Schaerer (1999) produced a detailed model of I Zw 18 and find that the photoionized model predicts a $T_{e}(\mathrm{O}$ III $)$ value $15 \%$ smaller than observed, on the other hand their model predicts an $I C F(\mathrm{He})=1.00$. From the observations of $\lambda \lambda 5876$ and 6678 by Izotov et al. (1999) of I Zw 18, and adopting a $T_{e}$ (He II) $10 \%$ smaller than $T_{e}$ (O III) we obtain $Y_{p}=0.237 \pm 0.007$; for a $T_{e}$ (He II) $15 \%$ smaller than $T_{e}\left(\mathrm{O}\right.$ III) we obtain $Y_{p}=0.234 \pm 0.007$, both results in good agreement with our determination based on the SMC. Further discussion of these issues is presented elsewhere (Peimbert, Peimbert, \& Luridiana 2000b).

The primordial helium abundance by mass of $0.2345 \pm 0.0030(1 \sigma)$ - based on the SMC - combined with the computations by Copi, Schramm, \& Turner (1995) for three light neutrino species implies that, at the 95 percent confidence level, $\Omega_{b} h^{2}$ is in the 0.0046 to 0.0103 range. For $h=0.65$ the $Y_{p}$ value corresponds to $0.011<\Omega_{b}<0.024$, a value considerably smaller than that derived from the pregalactic deuterium abundance, $D_{p}$, determined by Burles \& Tytler (1998) 
that corresponds to $0.040<\Omega_{b}<0.050$ for $h=0.65$, but in very good agreement with the observational estimate of the global budget of baryons by Fukugita, Hogan, \& Peebles (1998) who find $0.007<\Omega_{b}<0.038$ for $h=0.65$. The discrepancy between $Y_{p}$ and $D_{p}$ needs to be studied further.

To increase the accuracy of the $Y_{p}$ determinations we need observations of very high quality of as many $\mathrm{He}$ I lines as possible to derive $T_{e}(\mathrm{He} \mathrm{II}), N_{e}$ (He II), and $\tau(3889)$ self-consistently. We also need observations with high spatial resolution to estimate the $I C F(\mathrm{He})$ along different lines of sight.

It is a pleasure to acknowledge several fruitful discussions on this subject with: L. Carigi, V. Luridiana, B. E. J. Pagel, M. T. Ruiz, E. Skillman, G. Steigman, S. Torres-Peimbert, and S. Viegas.

\section{References}

Armour, M. H., Ballantyne, D. R., Ferland, G. F., Karr, J., \& Martin, P.G. 1999, PASP, 111, 1251

Benjamin, R. A., Skillman, E. D., \& Smits, D. P. 1999, ApJ, 514, 307

Burles, S., \& Tytler, D. 1998, ApJ, 507, 732

Carigi, L. 2000, ApJ, submitted

Carigi, L., Colín, P., Peimbert, M., \& Sarmiento, A. 1995, ApJ, 445, 98

Carigi, L., Colín, P., \& Peimbert, M. 1999, ApJ, 514, 787

Chiappini, C., Matteucci, F., \& Gratton, R. 1997, ApJ, 477, 765

Copi, C. J., Schramm, D. N., \& Turner, M. S. 1995, Science, 267, 192

Esteban, C., Peimbert, M., Torres-Peimbert, S., \& Escalante, V. 1998, MNRAS, 295,401

Esteban, C., Peimbert, M., Torres-Peimbert, S., \& García-Rojas, J. 1999, RevMexAA, 35, 85

Ferland, G. J. 1996, Hazy, a Brief Introduction to CLOUDY, Univ. of Kentucky Dept. of Phys. \& Astron. Internal Report

Fields, B. D., \& Olive, K. A. 1998, ApJ, 506, 177

Fukugita, M., Hogan, C. J., \& Peebles, P. J. E. 1998, ApJ, 503, 518

Izotov, Y. I., Chaffee, F. H., Foltz, C. B., Green, R. F., Guseva, N. G., \& Thuan, T. X. 1999, ApJ, 527, 757

Izotov, Y. I., \& Thuan, T. X. 1998, ApJ, 500, 188

Izotov, Y. I., Thuan, T. X., \& Lipovetsky, V. A. 1994, ApJ, 435, 647

Izotov, Y. I., Thuan, T. X., \& Lipovetsky, V. A. 1997, ApJS, 108, 1

Luridiana, V., Peimbert, M., \& Leitherer, C. 1999, ApJ, 527, 110

Maeder, A. 1992, A\&A, 264, 105

Massey, P., Parker, J. W., \& Garmany, C. D. 1989, AJ, 98, 1305

Pagel, B. E. J., Simonson, E. A., Terlevich, R. J., \& Edmonds, M. G. 1992, MNRAS, 255, 325

Peimbert, A., Peimbert, M., \& Luridiana, V. 2000b, RevMexAA, submitted

Peimbert, M. 1967, ApJ, 150, 825 
Peimbert, M. 1995, in The Analysis of Emission Lines., ed. R.E. Williams \& M. Livio (Cambridge University Press), p. 165

Peimbert, M., Peimbert, A., \& Ruiz, M.T. 2000a, ApJ, submitted

Peimbert, M., \& Torres-Peimbert, S. 1999, ApJ, 525 (Part 3), 1143

Peimbert, M., Torres-Peimbert, S., \& Ruiz, M.T. 1992, RevMexAA, 24, 155

Robbins, R. R. 1968, ApJ, 151, 511

Shields, G. A. 1974, ApJ, 191, 309

Stasinska, G., \& Schaerer, D. 1999, A\&A, 351, 72

Vílchez, J. M., \& Pagel, B. E. J. 1988, MNRAS, 231, 257

Woosley, S. E., Langer, N., \& Weaver, T. A. 1993, ApJ, 411, 823

Woosley, S. E., \& Weaver, T. A. 1995, ApJS, 101, 181 